\title{
Adaptation in motion perception: Alteration of motion evoked by ocular pursuit
}

\author{
JOSHUA BACON \\ Tufts University, Medford, Massachusetts 02155 \\ and \\ HANS WALLACH \\ Swarthmore College, Swarthmore, Pennsylvania 19081
}

\begin{abstract}
A new perceptual adaptation, an alteration in the perceived direction of motion given by ocular pursuit, is reported. When an object starts to move on a straight path, its displacement is initially given by a shift of its image on the retinas of the stationary eyes; then, after about $200 \mathrm{msec}$, the eyes start to track the moving object. The perception of motion that results from ocular pursuit was altered by causing ocular pursuit of a moving object to be preceded regularly by a displacement of the object's image whose direction differed from the direction of the pursuit movement. This was arranged by changing the direction of the given motion at approximately the moment when image displacement changed into tracking. Prolonged exposure to such conditions resulted in a change of the tracked motion's apparent direction, which became somewhat more like the direction of the preceding motion phase that was given by image displacement.
\end{abstract}

One route to the discovery of a new perceptual adaptation starts with a consideration of acquired perceptual cues (i.e., those that have become stimuli through a learning process), since they are more likely than innate perceptual cues to yield to an adaptation procedure. That was our course in the present instance. Of the three conditions of stimulation known to evoke motion perception in the frontal plane-displacement of the image of the moving object on the retina, ocular pursuit of the moving object, and change of configuration resulting from the displacement of the moving object relative to its context-only the first one seems likely to be innate. Wallach $(1976$, p. 98$)$ has argued that only image displacement is a primary condition of stimulation for motion perception, whereas configurational change and ocular pursuit are learned. The latter occur whether or not they function as stimuli for motion perception. Configurational change is very frequently present when a moving object is observed, either with stationary objects visible at the same time or against a stationary background pattern. In either case, the displacement of the object relative to its visual surround is given as changing configuration. Ocular pursuit of the moving object would also take place if it did not produce motion perception; it is needed to provide nearly stationary

This work was supported by Grant BNS 7924462 from the National Science Foundation to Swarthmore College, Hans Wallach principal investigator. We are most grateful to Martha Teghtsoonian for her help in writing this report. images of moving objects, a condition that favors recognition. Presumably, learning turns existing conditions into stimuli that evoke motion perception. It is easy to see how this might happen in the case of configurational change. Often when a moving object is faced, there is a displacement of the moving object relative to a surround that makes itself felt as configurational change. Simultaneously, other cues for motion perception-ocular pursuit when the eyes track the moving object or image displacement when they rest on a part of the stationary surround-cause perceived motion of the object. Frequent simultaneity of configurational change with perceived motion may establish a relation between them and make configurational change a stimulus for perceived motion in such a way that the amount of perceived motion comes to vary with the amount of configurational change.

Wallach, Bacon, and Schulman (1978) were able to demonstrate that the perception of motion that results from configurational change can be altered by perceptual adaptation. They did this by applying the often-used method of creating a cue conflict. The stimulation for a perceptual variable such as visual direction, distance, or depth is altered by some interposed device. A conflict between the altered stimulation and other stimuli for the same variable results, and prolonged exposure to this stimulus discrepancy brings about a change in the relation between the altered stimulation and the perception it evokes. In the case of configurational change, however, no interposed device was needed, because its 
peculiar nature results, under certain conditions, in nonveridical motion perception. Normally, configurational change occurs when the motion of an object results in a relative displacement between the object and its stationary surround, which causes the object's motion to be perceived. But the same relative displacement and the same configurational change may occur when the object is stationary and the surround moves, and it then often has the same perceptual result: the object appears to move. This nonveridical motion is called induced motion, and it occurs in spite of the presence of another motion cue, image displacement, which represents the object correctly as stationary. Wallach et al. (1978) obtained adaptation by exposing the subject to a condition in which a stationary object was perceived in continuous induced motion but in which the absence of image displacement represented the object as stationary. The extent of the perceived induced motion was, on the average, $15 \%$ smaller after a 10-min exposure than it had been before.

We made an attempt to obtain an alteration of the direction of the perceived motion evoked by ocular pursuit in an analogous fashion-namely, by creating a cue conflict. The subject had to track a dot presented in reciprocating vertical motion, seen against a background pattern that moved horizontally in reciprocating motion, in phase with the motion of the dot. The two motions resulted in an oblique relative displacement between the dot and the background pattern, which was given as configurational change. This oblique configurational change and the ocular pursuit of the vertically moving dot formed the cue conflict to which the subject was exposed during the attempted adaptation. Before and after the 10-min exposure period, the subject gave estimates of the direction of the motion of the tracked spot in the absence of the background pattern. Although, during exposure, configurational change prevailed over pursuit and the subject saw the dot move obliquely, no adaptation resulted: The direction of the perceived motion evoked by pursuit did not change.

Eventually, we arrived at a successful adaptation condition by taking a very different approach. We reasoned that, if we knew the condition under which ocular pursuit becomes a stimulus for perceived motion, we might use an analogous condition to teach the subject a relation between pursuit and perceived motion different from the normal one and obtain a perceptual change that looks like adaptation. Earlier, one of us (Wallach, 1976) proposed that pursuit eye movements become stimuli for motion perception because they are frequently paired with image displacement. Pursuit eye movements are preceded by brief periods during which the motion of the object that is subsequently tracked is given by a displacement of its retinal image. "This regular se- quence of events would favor a learning process through which the capacity to evoke motion perception is transferred from image displacement to pursuit movement"' (p. 98). If this were the manner in which pursuit movements become stimuli for motion perception, exposure to an alteration of this sequence might lead to an altered motion perception.

To be more specific: When a stationary object in a homogeneous surround starts to move, its image is initially displaced on the retina for a brief period and then the eyes follow it. When the object moves straight, as usually happens, the two sequential stimulation phases, image displacement and pursuit, have the same direction. If it were true that the capacity of pursuit movements to evoke motion perception is learned, and if a particular perceived motion resulted from a pursuit movement because this movement was previously associated with an image displacement of the same direction, it may be possible to alter the motion perception that results from pursuit by altering that stimulation sequence. We arranged for the motion given by pursuit to have a direction different from the motion given by image displacement and presented the same new phase sequence a large number of times during the exposure period. In our experiments, the pursuit phase was oblique; the tracked target moved upward on a 45-deg slope. Ordinarily, this pursuit phase would be preceded by a period lasting approximately $200 \mathrm{msec}$ during which the oblique target motion is given as an image displacement (Robinson, 1965). We arranged for the target motion that is given as image displacement to be horizontal so that the subject is repeatedly exposed to a sequence in which an oblique target motion largely given by ocular pursuit is preceded by a brief phase in which horizontal motion of the same target is given, at least in part, by image displacement. If the pairing of the oblique pursuit phase with a preceding horizontal image displacement comes to have an effect on the perceived direction of motion in which the pursuit phase results, the perceived direction of the target should appear somewhat more horizontal after exposure to the adaptation conditions than before. The following experiments show that such an adaptation can be obtained.

\section{EXPERIMENT 1}

\section{Method}

Subjects. Twenty-three paid undergraduates of Tufts University served as subjects, 11 in the experimental condition and 12 in the control condition.

Apparatus. The moving object (target) was a small cross $(3 \times 3 \mathrm{~mm})$ displayed on a Tektronics Model 600 oscilloscope. It was generated by a PDP 8/e computer of the Digital Equipment Corporation. A pair of electrodes signaled the beginning of the eye movement in each trial. One electrode was fastened to the outer orbital ridge of the right eye and the other, to the bridge of the nose. The signals from the electrodes were amplified 
so that an eye movement of $.25 \mathrm{deg}$ would trigger the computer to change the target motion, with the proper amplification set for each subject. To insure that only the moving target and no afterglow was seen, the subject wore goggles with neutral density filters. The subjects gave estimates of the slope angle of the second, oblique phase by moving one leg of a pair of rods that were joined at their ends by a bolt under spring tension. One leg of the rod angle was fastened in horizontal position to the edge of a table so that half of it protruded beyond it.

Procedure. There were four parts to the procedure-a preadaptation test, an adaptation period, an immediate postadaptation test, and a delayed postadaptation test. During all four parts, the subject initiated a trial by pressing a button; he or she was under instructions to look at the target as soon as it appeared and to keep her or his eyes on it until it had disappeared at the end of each trial. The subject viewed the motion display in a darkened room.

When the target first appeared it was at the center of the display and directly in front of the subject and at his eye level. It remained stationary for a period of time ranging from .5 to $1.5 \mathrm{sec}$ and then started to move horizontally to the right at a constant velocity of $5.12 \mathrm{~cm} / \mathrm{sec}$ or $12.6 \mathrm{deg} / \mathrm{sec}$ of visual angle. When the subject moved his eyes $.25 \mathrm{deg}$, the computer changed the direction of the target motion upward by one of three angles, and the target moved $6 \mathrm{~cm}$ in the new direction before it disappeared. In the tests, the direction change was either 10 or $25 \mathrm{deg}$; in the adaptation period, it was $45 \mathrm{deg}$. During the oblique phase, the velocity of the horizontal component of the oblique motion remained the same, $5.12 \mathrm{~cm} / \mathrm{sec}$. Thus, the velocities of the oblique motion were 5.2 and $5.65 \mathrm{~cm} / \mathrm{sec}$ in the tests and $7.24 \mathrm{~cm} / \mathrm{sec}$ during adaptation.

The preadaptation test consisted of six trials, three for the motion sequences with a 10-deg change and three with a 25 -deg change, given in alternation. The subject's task was to change the slope of the movable leg of the rod angle so that it matched the slope of the second phase of the apparent path of the target. This was done in the dark, with the subject feeling along the rods. During each trial, the subject was shown the motion sequence four times and was permitted to set the leg or change its position after each observation, the last of which was recorded as a slope estimate.

During the adaptation period, the change in the motion direction was always $45 \mathrm{deg}$. The adaptation period consisted of 75 presentation sequences and lasted, on the average, $12 \mathrm{~min}$. If, during the initial fixation period during which the target was stationary, the subject moved his eyes too soon (i.e., before the target started to move horizontally), the computer canceled that presentation sequence and reset for a new one. The typical number of such aborted trials was 10 and never exceeded 30 .

The postadaptation test consisted of two trials only, one with the 10-deg change and the other with the 25-deg change, in the same order as the first two trials in the preadaptation test. After the subject had given these two estimates, he sat for $10 \mathrm{~min}$ with closed eyes, and then the two test trials were given again. The second test was given to find out whether the adaptation effect would decrease during a lapse of time that approximated the duration of the adaptation period.

It should be emphasized that the filters that the subjects wore ensured that only the target was visible. Therefore, its motion was given either by image displacement or by pursuit. Also, because the subject was under instruction to keep his eyes on the target, which was stationary at the start of each test or exposure trial and began to move after a variable time interval, and because the electrode signal showed when the eyes were at rest and when they moved, we know that there was a brief period at the beginning of each trial when the initial horizontal motion of the target was given as a horizontal image displacement. The duration of this image displacement varied between 230 and $280 \mathrm{msec}$, and the extent of the image displacement corresponded to a visual angle of 2.9 to $3.5 \mathrm{deg}$. This approximate duration range was known from the time interval between the beginning of the target motion and the time point at which the motion direction changed. This interval was registered by the computer and ranged from 250 to $300 \mathrm{msec}$. It consisted of the sum of the duration of the image displacement and of the duration of the .25 -deg eye movement that triggered the computer to change the target motion; the latter probably amounted to $20 \mathrm{msec}$.

To make sure that the change in the perception of the pursuit phase of the target motion that we expected to occur was indeed the result of the horizontal image displacement phase that preceded it, we did a control experiment in which the latter was omitted in the adaptation period. Instead, a presentation consisted of an unchanging target motion of 45-deg slope, which resulted, of course, in an oblique image displacement phase followed by a pursuit phase of the same direction. Here, too, the number of presentations during exposure was 75 . Due to a programming error, the tests contained only one of the motion sequences from the experimental condition, the sequence with the 25 -deg direction change. The other sequence had a 15-deg change.

\section{Results}

The means of the estimated slope angles are listed in the upper part of Table 1, together with their standard deviations. The preadaptation scores were averages of the three individual slope estimates obtained for a motion sequence. The scores of the other means consisted of single estimates. After exposure, the mean estimates of the slope angle were considerably smaller in the experimental condition than before-by $12.4 \mathrm{deg}$ when the change of the target motion in the test was $25 \mathrm{deg}$ and by $8.8 \mathrm{deg}$ when that change was $10 \mathrm{deg}$, and both differences were highly significant $[\mathrm{t}(10)=5.60$ and 4.91 , respectively].These results differed from those of the control group, in which the exposure period did not produce significant changes of the perceived slope angle either for a $15-$ or $25-\mathrm{deg}$ motion path. The change in the mean slope estimates measured with the 25-deg target motion in the experimental condition was significantly different from the corresponding change in the control condition $[\mathrm{t}(21)=4.05]$.

\section{EXPERIMENT 2}

Our second experiment was a simpler version of the experiment just reported and shows how easily the adaptation effect can be obtained. The change in the direction of the target motion was no longer triggered by a signal produced by the start of the subject's eye movement. Instead, the direction change always occurred $195 \mathrm{msec}$ after the beginning of the target motion. Second, the direction changes during adaptation and in the test presentations were no longer different. Instead, the same direction change was presented during exposure and in the tests. After the initial horizontal motion phase, the target motion always formed an angle of $22 \mathrm{deg}$ with the horizontal. In the control condition, the target motion had a uniform slope of $22 \mathrm{deg}$. There were two 
further changes. The observation distance that had been $23 \mathrm{~cm}$ in Experiment 1 was increased to $60 \mathrm{~cm}$. This changed the angular velocity from 12.6 to 4.9 $\mathrm{deg} / \mathrm{sec}$. Finally, the time interval between the first and second postadaptation tests was shortened from 10 to $5 \mathrm{~min}$. Experimental and control conditions each had 16 subjects.

\section{Results}

The means of the slope angle estimates are shown in the second part of Table 1. As in Experiment 1, the mean slope angle in the experimental condition was significantly smaller immediately after the adaptation exposure than before $[\mathrm{t}(15)=3.73]$. Immediately after the exposure period in the control condition, in which the initial horizontal motion phase was omitted, the small change was not significant. The mean differences between pre- and postexposure estimates were 5.5 and $1.0 \mathrm{deg}$ for experimental and control conditions, respectively; that difference was significant at the .02 level $[\mathrm{t}(30)=2.43]$.

The effect of the dissipation periods. When, in Experiment 2, slope estimates were obtained after a time lapse of $5 \mathrm{~min}$, their means were virtually unchanged from those of the immediate postadaptation estimates, although the variance of the estimates had increased. The difference between means for the preadaptation and the delayed postadaptation estimates was still significant $[\mathrm{t}(15)=2.79, \mathrm{p}<.02]$. The effect of the adaptation exposure in the experimental condition had apparently not dissipated.

In Experiment 1, when the dissipation period lasted $10 \mathrm{~min}$, the adaptation effect clearly diminished during that delay. For the $25-$ deg direction change, both postadaptation means, the immediate and the delayed one, were significantly different from the preadaptation mean, but the mean difference score $(A-B)$ between the preadaptation and the immediate postadaptation scores was more than twice as great as the difference $(A-C)$ between the preadaptation scores and the delayed postadaptation scores, and that difference was also significant $[t(10)=2.28$, $\mathrm{p}<.05$ ]. For the 10-deg direction change, no adaptation effect remained after the 10-min delay period.

\section{DISCUSSION}

We have discovered a new kind of perceptual adaptation using a novel approach. However, that does not mean that the manner in which we obtained a change in the relation between the direction of ocular pursuit and the direction of the resulting perceived motion does not fit the cue conflict paradigm, which applies to at least four of the known kinds of perceptual adaptation. Adaptation based on cue conflict may take place whenever more than one kind of stimulation serves as a cue for the same perceptual variable, a ubiquitous occurrence in space perception and, as we have reported, in motion perception also. When one of the "paired" cues is altered by means of an interposed device, while another cue represents the given objective variable veridically, a cue conflict exists. If adaptation develops and the perceptual process that results from the altered cue changes in such a way that its result becomes less different from the perception resulting from the veridical cue than was initially the case, the cue conflict is diminished. If such a change takes place, the perception resulting from the altered cue, in effect, compensates, in part or totally, for the cue-altering effect of the interposed device.

Adaptation in stereoscopic depth perception (Wallach, Moore, \& Davidson, 1963) may serve as an example. The interposed device consisted here of an arrangement of small mirrors that increased binocular parallax and caused the depth of threedimensional wire forms to be given with enhanced disparities. Therefore, the ensuing process, stereoscopic depth perception, resulted in an experience of wire forms that were too large in the depth dimension. This deformation could be measured by obtaining an estimate of the depth of the wire form.

Table 1

Mean Slope Estimation Scores and Standard Deviations (in Degrees) for Pursuit Phase Before and After Exposure and After Dissipation Period

\begin{tabular}{|c|c|c|c|c|c|c|c|c|c|c|c|}
\hline \multirow[b]{2}{*}{ Group } & \multirow[b]{2}{*}{ Test Condition } & \multicolumn{2}{|c|}{$\mathbf{A}$} & \multicolumn{2}{|c|}{ B } & \multicolumn{2}{|c|}{$\mathbf{A}-\mathbf{B}$} & \multicolumn{2}{|c|}{$\mathrm{C}$} & \multicolumn{2}{|c|}{$A-C$} \\
\hline & & Mean & SD & Mean & SD & Mean & $\mathrm{SD}$ & Mean & SD & Mean & SD \\
\hline & & \multicolumn{10}{|c|}{ Experiment $1(\mathrm{Ns}=11$ and 12$)$} \\
\hline Experimental & $\begin{array}{l}\text { 10-Deg Change } \\
\text { 25-Deg Change }\end{array}$ & $\begin{array}{l}15.4 \\
32.3\end{array}$ & $\begin{array}{l}7.1 \\
6.3\end{array}$ & $\begin{array}{r}6.6 \\
20.0\end{array}$ & $\begin{array}{l}5.8 \\
9.2\end{array}$ & $\begin{array}{r}8.8 \\
12.4\end{array}$ & $\begin{array}{l}7.0 \\
7.3\end{array}$ & $\begin{array}{l}13.5 \\
26.9\end{array}$ & $\begin{array}{r}7.0 \\
10.1\end{array}$ & $\begin{array}{l}1.9 \\
5.5\end{array}$ & $\begin{array}{l}7.2 \\
7.1\end{array}$ \\
\hline \multirow[t]{2}{*}{ Control } & $\begin{array}{l}\text { 15-Deg Change } \\
\text { 25-Deg Change }\end{array}$ & $\begin{array}{l}22.3 \\
32.9\end{array}$ & $\begin{array}{l}4.5 \\
7.3\end{array}$ & $\begin{array}{l}20.6 \\
31.5\end{array}$ & $\begin{array}{l}6.7 \\
9.6\end{array}$ & $\begin{array}{l}1.7 \\
1.5\end{array}$ & $\begin{array}{l}5.6 \\
5.3\end{array}$ & $\begin{array}{l}23.2 \\
34.0\end{array}$ & $\begin{array}{l}8.0 \\
9.6\end{array}$ & $\begin{array}{l}-.9 \\
1.1\end{array}$ & $\begin{array}{l}7.5 \\
7.1\end{array}$ \\
\hline & & \multicolumn{10}{|c|}{ Experiment $2(\mathrm{Ns}=16$ and 16$)$} \\
\hline $\begin{array}{l}\text { Experimental } \\
\text { Control }\end{array}$ & $\begin{array}{l}\text { 22-Deg Change } \\
\text { 22-Deg Change }\end{array}$ & $\begin{array}{l}30.2 \\
24.6 \\
\end{array}$ & $\begin{array}{l}6.8 \\
8.3\end{array}$ & $\begin{array}{l}24.6 \\
23.6 \\
\end{array}$ & $\begin{array}{l}6.8 \\
7.5\end{array}$ & $\begin{array}{l}5.5 \\
1.0\end{array}$ & $\begin{array}{l}5.9 \\
4.5\end{array}$ & $\begin{array}{l}25.1 \\
23.8\end{array}$ & $\begin{array}{l}9.2 \\
8.3\end{array}$ & $\begin{array}{r}5.1 \\
.8\end{array}$ & $\begin{array}{l}7.2 \\
4.6 \\
\end{array}$ \\
\hline
\end{tabular}

Note $-A=$ preadaptation $; B=$ immediate postadaptation $; C=$ delayed postadaptation. 
Stimulation representing veridical depth was provided by having the wire form rotate about a vertical axis. The resulting kinetic depth effect would normally produce the perception of the correct wire form in rotation. But, seen through the mirror arrangement, the rotating wire form seemed to be in incessant distortion. A cross section that occupied the frontal plane and was correctly perceived would soon be oriented in the sagittal direction, and the interposed device caused it to appear stretched in depth, and so on. A $10-\mathrm{min}$ exposure to viewing a slowly rotating wire form through the mirror arrangement resulted in partial compensation for the artificially enhanced disparity; the deformation of the wire form caused by the mirror arrangement decreased. As a result, the apparent distortion that the rotating form seemed to undergo diminished, and estimates of the depth of the stationary wire form became smaller.

To compare our experiments with adaptation in stereoscopic depth perception, imagine that a device existed that altered the stimulation caused by a straight motion path so that it corresponded to a motion path of different direction, but that the device was such that only ocular pursuit was affected, while image displacement was not altered. Were a horizontal motion to be viewed through such a device, the same sequence of stimulation would result as the one we presented during the exposure period; the given motion direction would change contingent on the start of ocular pursuit. Thus, the device would provide deceptive stimulation when an objective motion is given by pursuit and veridical stimulation when it is given as image displacement. If there were adaptation to this device (i.e., if eventually perception partially compensated for its effect), perceived motion resulting from ocular pursuit would become less oblique, as it did in our experiment. The condition that forced oblique pursuit in the exposure period provided the altered cue and corresponded to the enhanced disparity in adaptation in stereoscopic depth perception, and the image displacement phase in our experiment corresponded to the retinal projection of the rotation of the wire form that provided the veridical stimulation. There was one difference: Rotation and enhanced disparities were simultaneous, while image displacement and pursuit were successive, but, apart from that, the cue conflict paradigm holds for our experiment.

An additional fact connected with the succession of the two motion phases must be considered. The motion given by image displacement covered approximately $1 \mathrm{~cm}$, while the motion that occurred after the eyes had started to move covered $6 \mathrm{~cm}$ and lasted about four times as long as the former. The fact that strong adaptation developed in a brief timein spite of this imbalance between the veridical stimulation and the stimulation whose effect was changed by adaptation-suggested to us that adaptation resulted here from associative learning. The image displacement phase resulted in perception of a horizontal motion, and the contiguity between the oblique pursuit movements and the perceived horizontal motion resulted in formation of associations between them that competed with the result of previous learning responsible for perceived oblique motion resulting from oblique pursuit. A temporary effectiveness of the associations formed during exposure would account for our test results.

\section{REFERENCES}

Rosinson, D. A. The mechanism of human smooth pursuit eye movement. Journal of Physiology, 1965, 180, 569-591.

Wallach, H. On perception. New York: Quadrangle/The New York Times Book Co., 1976.

Wallach, H., Bacon, J., \& Schulman, P. Adaptation in motion perception: Alteration of induced motion. Perception \& Psychophysics, 1978, 24, 509-514.

Wallach, H., Moore, M. E., \& Davidson, L. Modification of stereoscopic depth perception. American Journal of Psychology, 1963, 76, 191-204.

(Manuscript received May 4, 1981; revision accepted for publication November 3, 1981.) 